CASE REPORT

\author{
L. Legrand \\ L. Catherine \\ F. Brivet \\ D. Musset
}

\section{Solitary Hypothalamopituitary Toxoplasmosis Abscess in a Patient with AIDS}

SUMMARY: Toxoplasmosis is a disease caused by an obligate intracellular protozoal parasite, Toxoplasma gondii. It is the most common cause of focal brain lesions in patients with AIDS. The imaging features and endocrine disorders of CNS toxoplasmosis in patients with AIDS are reviewed.

ABBREVIATIONS: CNS $=$ central nervous system; FLAIR $=$ fluid-attenuated inversion recovery; $\mathrm{HIV}=$ human immunodeficiency virus; $\mathrm{PCR}=$ polymerase chain reaction
$\mathbf{T}$ oxoplasmosis is the most common cause of focal brain lesions in patients with AIDS, ${ }^{1}$ even if the number of cases has declined with the introduction of highly active antiretroviral therapy. Neuroimaging usually reveals multiple nodular or ring-enhancing lesions with edema and mass effect. ${ }^{2}$ The clinical manifestations are nonspecific and depend on the location of the lesions. Focal neurologic symptoms are often superimposed on global encephalopathy. Infections of endocrine organs result in endocrine disorders. We present a unique case of solitary hypothalamopituitary toxoplasmosis abscess causing central diabetes insipidus and corticotropic insufficiency and revealing AIDS.

\section{Case Report}

A 36-year-old woman consulted us for fever and pain in the maxillary area. She was treated with amoxicillin, clavulanate potassium, and corticotherapy for 8 days for suspicion of sinusitis. Persistence of fever led to another antibiotic treatment (levofloxacin) for 9 days. Seven days later, she was admitted to the emergency department for confusion, hypothermia, and hypotension.

Unenhanced CT revealed a $15-\mathrm{mm}$ area of abnormal low attenuation centered on the optic chiasm and hypothalamic region. This area demonstrated ring enhancement on postcontrast CT images. MR imaging confirmed the single character of the lesion, which was hyperintense on T2-weighted sequences and surrounded by high-signal-intensity vasogenic edema involving the optic tract, cerebral peduncles, anterior commissure, internal parts of the temporal lobes, and posterior arms of the internal capsules. The lesion was isointense on T1-weighted sequences, with an asymmetric target sign (a small eccentric nodule along the wall of the enhancing ring) on postcontrast sequences. ${ }^{3}$ The pituitary stalk was thickened (Fig 1).

A rapid screening test was positive for HIV. Examination of blood revealed lymphopenia and $8 \mathrm{CD} 4$ cells $/ \mathrm{mm}^{3}$. Serum toxoplasma immunoglobulin $\mathrm{G}$ was positive. Testing of CSF revealed no pleocytosis but elevated levels of protein, hypoglycorrhachia, and a PCR positive for Toxoplasma gondii.

Treatment was started, including pyrimethamine, sulfadiazine,

Received March 9, 2010; accepted March 15.

From the Departments of Radiology (L.L., L.C., D.M.) and Medical Reanimation (F.B.), Antoine Beclere Hospital, Clamart, France.

Please address correspondence to Laurence Legrand, MD, Department of Radiology, Antoine Beclere Hospital, 157 rue de la Porte de Trivaux, 92140 Clamart, France; e-mail: legrand.lau@gmail.com

DOI 10.3174/ajnr.A2139 and folinic acid. In addition, the patient received antibiotics for 3 days on the basis of the hypothesis of pyogenic abscess.

At day 3, the patient developed polyuria and polydipsia. Natremia and plasma osmolality were high whereas urine osmolality was low. Response to vasopressin led to the diagnosis of diabetes insipidus. Endocrine testing was performed, revealing a corticotropic insufficiency, which was treated with hydrocortisone. The hypothalamothyroid axis was not disturbed. Ophthalmologic examination revealed quadrantanopia.

Control MR imaging performed 3 weeks after the beginning of the treatment demonstrated almost complete regression, with nothing but a small area of high signal intensity on T2-weighted images and a light enhancement of the hypothalamic region (Fig 2). The clinical outcome confirmed the diagnosis of CNS toxoplasmosis.

\section{Discussion}

To our knowledge, this is the first report of unique hypothalamopituitary toxoplasmosis abscess responsible for central diabetes insipidus and corticotropic insufficiency.

$T$ gondii can infect any cell in the brain, but most lesions occur in the basal ganglia, thalamus, and corticomedullary junction. Lesions are usually multiple and are solitary in only approximately $14 \%$ of cases. ${ }^{3}$ A solitary lesion in a patient with AIDS is more likely to be a lymphoma (the second most common cause of space-occupying brain lesions in AIDS) than toxoplasmosis, ${ }^{4}$ and imaging appearances can be very similar. Several techniques have been suggested to help differentiate both, including diffusionweighted imaging, ${ }^{5}$ MR spectroscopy, single-photon emission CT, and positron-emission tomography. ${ }^{2}$ However, none of these has high specificity, and they are only useful when combined.

Also, a solitary brain abscess can be caused by other opportunistic microorganisms, such as Mycobacterium species, Aspergillus species, Nocardia species, Cryptococcus neoformans, and Listeria monocytogenes. Therefore, the presence of an intracranial mass in a patient with AIDS allows empiric treatment for cerebral toxoplasmosis, and failure to respond to therapy within the first 2 weeks dictates the need for a stereotactic biopsy. ${ }^{6,7}$ Although a definitive diagnosis of CNS toxoplasmosis relies on the identification of parasites by histopathology, the clinical and radiologic data can be complemented by a less invasive approach that confirms the presence of parasites in the CSF. In a recent study, a positive PCR as in our case has been found to have high sensitivity and specificity (83.3\% and $97.5 \%$, respectively). ${ }^{8}$

Opportunistic infection is one of the various pathogenic 

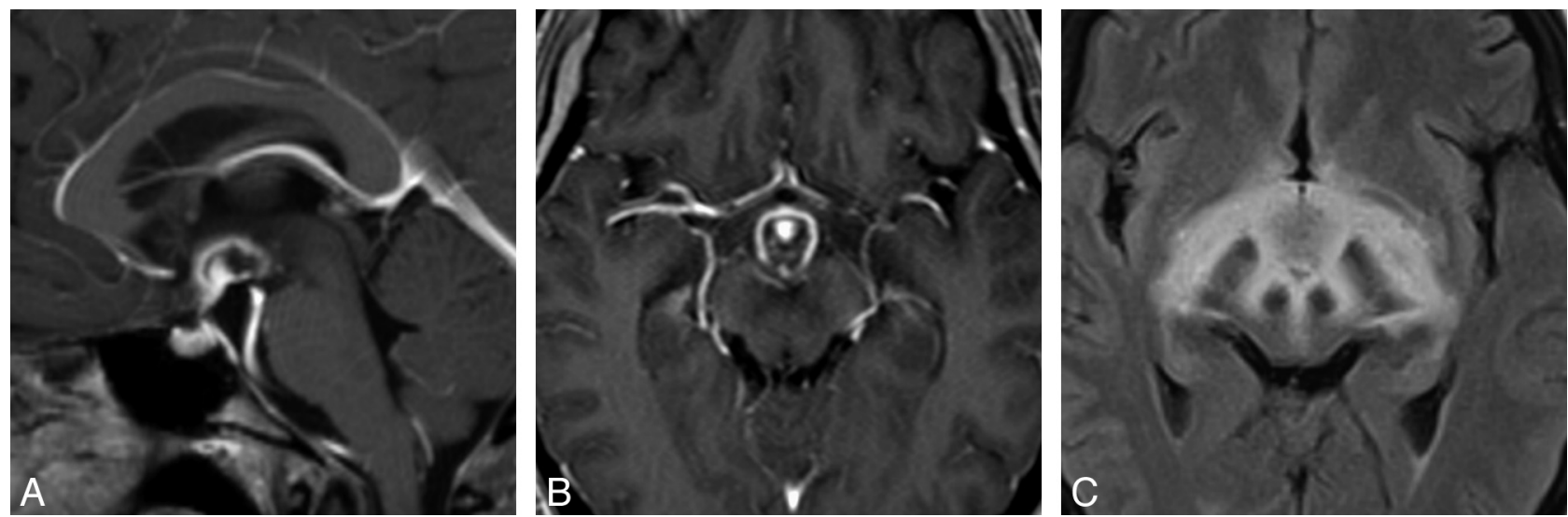

Fig 1. MR imaging at diagnosis. A, Sagittal contrast-enhanced T1-weighted image shows a ring-enhancing lesion of the hypothalamopituitary axis. $B$, Axial contrast-enhanced T1-weighted image shows the lesion centered on the hypothalamus and the optic chiasm. $C$, Axial FLAIR image demonstrates marked vasogenic edema that involves the hypothalamus and mesencephalon
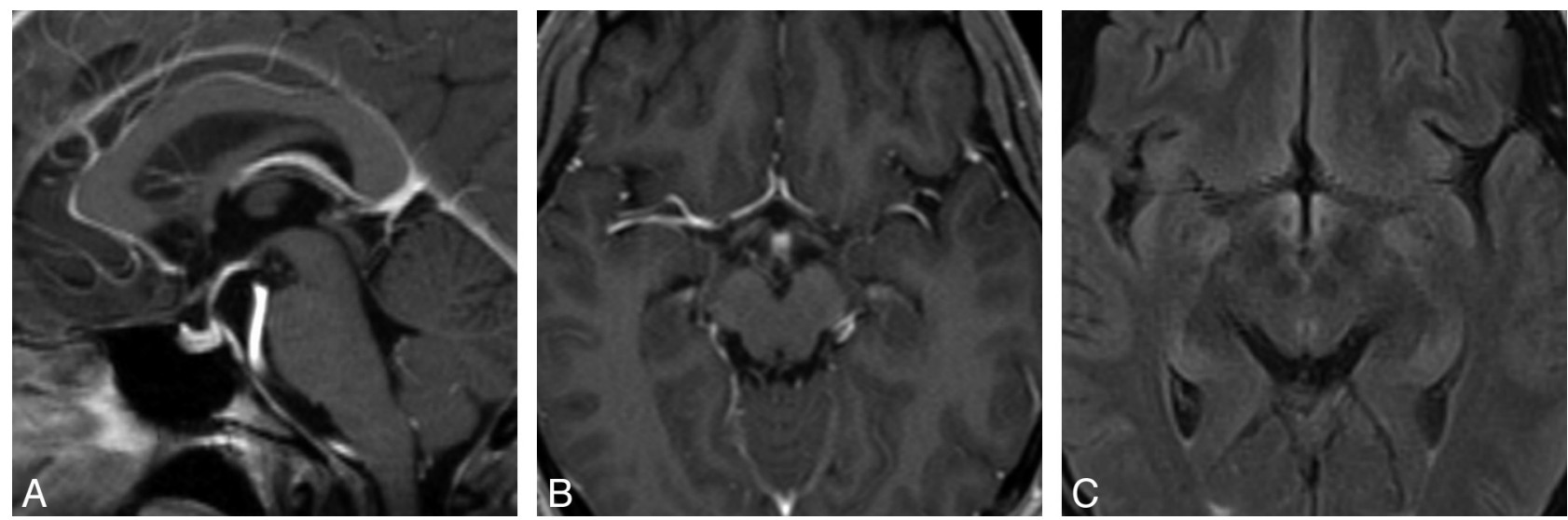

Fig 2. MR images obtained after treatment. $A$ and $B$, Sagittal and axial contrast-enhanced T1-weighted images show complete regression of the abscess. $C$, Axial FLAIR image demonstrates a nearly complete regression of the edema.

mechanisms leading to endocrinopathies of the HIV disease. ${ }^{9}$ Others include neoplasms, drugs administered to treat infections, and cytokine abnormalities associated with the HIV disease process. ${ }^{10}$ There have been a few cases of diabetes insipidus, ${ }^{11-14}$ panhypopituitarism, ${ }^{15}$ or both ${ }^{11}$ complicating CNS toxoplasmosis among patients with AIDS. In these reports, cerebral imaging findings were either normal (earlier cases $^{12,13}$ ) or showed multiple abscesses as usually described in cerebral toxoplasmosis, some involving the pituitary gland ${ }^{15}$ or the infundibulum (pituitary stalk). ${ }^{11}$

\section{Conclusions}

CNS toxoplasmosis can have unusual presentations in patients with AIDS, whether it is the solitary nature of the abscess or its rare localization. When hypothalamopituitary dysfunction complicates the infection, antiparasitic medication is effective in the treatment of endocrine abnormalities.

\section{References}

1. Wright D, Schneider A, Berger JR. Central nervous system opportunistic infections. Neuroimaging Clin N Am 1997;7:513-25

2. Berger JR. Mass lesions of the brain in AIDS: the dilemmas of distinguishing toxoplasmosis from primary CNS lymphoma. AJNR Am J Neuroradiol 2003; 24:554-55

3. Smith AB, Smirniotopoulos JG, Rushing EJ. From the archives of the AFIP: central nervous system infections associated with human immunodeficiency virus infection-radiologic-pathologic correlation. Radiographics 2008;28: 2033-58

4. Ciricillo SF, Rosenblum ML. Imaging of solitary lesions in AIDS. J Neurosurg 1991;74:1029

5. Camacho DL, Smith JK, Castillo M. Differentiation of toxoplasmosis and lymphoma in AIDS patients by using apparent diffusion coefficients. AJNR Am J Neuroradiol 2003;24:633-37

6. Collazos J. Opportunistic infections of the CNS in patients with AIDS: diagnosis and management. CNS Drugs 2003;17:869-87

7. Araujo-Filho Sda C, Maia L, Silva HB, et al. Mesencephalic tuberculous abscess in a patient with AIDS. Arq Neuropsiquiatr 2008;66:259-60

8. Alfonso Y, Fraga J, Fonseca C, et al. Molecular diagnosis of Toxoplasma gondii infection in cerebrospinal fluid from AIDS patients. Cerebrospinal Fluid Res 2009;6:2

9. Grinspoon SK, Bilezikian JP. HIV disease and the endocrine system. N Engl J Med 1992;327:1360-65

10. Zapanti E, Terzidis K, Chrousos G. Dysfunction of the hypothalamic-pituitaryadrenal axis in HIV infection and disease. Hormones (Athens) 2008;7:205-16

11. Brandle M, Vernazza PL, Oesterle M, et al. Cerebral toxoplasmosis with central diabetes insipidus and panhypopituitarism in a patient with AIDS [in German]. Schweiz Med Wochenschr 1995;125:684-87

12. Bernard PM, Fenasse R, Zafiropoulo A, et al. Diabetes insipidus due to acquired toxoplasmosis [in French]. Mars Med 1969;106:173-74

13. Santucci A, Aguglia F, Federico M, et al. Neuroendocrine syndromes with toxoplasmic origin: clinical and therapeutic considerations. Clin Ter 1980;94:3-19

14. Sanchez JF, Olmedo MC, Pascua FJ, et al. Diabetes insipidus as a manifestation of cerebral toxoplasmosis in an AIDS patient. Rev Neurol 2000;30:939-40

15. Milligan SA, Katz MS, Craven PC, et al. Toxoplasmosis presenting as panhypopituitarism in a patient with the acquired immune deficiency syndrome. Am J Med 1984;77:760-64 